\title{
Roadblocks to Implementing Modern Digital Infrastructure: Exploratory Study of API Deployment in Large Organizations
}

\author{
Nigel P. Melville \\ University of Michigan \\ npmelv@umich.edu
}

\author{
Rajiv Kohli \\ College of William and Mary \\ rajiv.kohli@mason.wm.edu
}

\begin{abstract}
Application programming interfaces (APIs) are an important component of digital infrastructure. Extensively employed in diverse industries, APIs are a boundary resource that enables new business models, enhances efficiency, and generates new sources of revenue. As little is known about how organizations deploy APIs, we conducted an exploratory examination of organizational deployment challenges of this important component of digital infrastructure. Analysis of semi-structured interview data collected within two large organizations reveals managerial challenges involving data, incentives, shared knowledge, and supplier management. Overall, our study contributes to knowledge about boundary resources while informing management practice concerning this emergent business imperative in the fourth industrial revolution.
\end{abstract}

\section{Introduction}

Digital infrastructure has been defined as "shared, unbounded, heterogeneous, open, and evolving sociotechnical systems" involving information technologies and associated capabilities, processes, and communities [12:1]. Application programming interfaces (APIs) are an important component of digital infrastructure due to their role as boundary resources interconnecting different systems. Digital infrastructure is important as it enables the provision of valuable digital services $[4,8]$. As such, APIs as a form of digital infrastructure are integral in the fourth industrial revolution by providing a digital "highway system" upon which generativity and innovation can thrive [23].
Digital infrastructure has been analyzed in the information systems (IS) literature from various perspectives, including competitiveness and firm growth [28] and generativity [13]. Moreover, several contexts have been examined, such as smart mobility [19], global payments [10], and international trade [16].

Boundary resources are a specific dimension of digital infrastructure important to platforms and innovation in large organizations [5]. By following established data exchange standards, API boundary resources expose data and resources to other systems within and across organizations to better align organizational objectives with digital resources. The importance of APIs in digital infrastructure has been emphasized in the literature: "APIs have moved to the front and center of the discussion on digital infrastructure and digital platforms over the last few years." [14:5306].

Evolving from earlier connecting technologies such as Extensible Markup Language (XML), modern APIs are unique as they enable web scale software reuse and resource sharing via simple, standardized protocols such as JavaScript Object Notation (JSON) and Representational State Transfer (REST) [17, 29].

APIs can be viewed through the lens of the strategic asset model view of digital infrastructure, in which "managers initiate and implement changes in an organization's portfolio of systems and tools for increasing the alignment between its information technology resources and strategic imperatives."[13:910]. Examples abound.

APIs provide an underlying digital infrastructure on which Walgreens has pursued a stream of digital business innovations - such as digital prescription fulfillment via third-party mobile health apps - and 
thereby supports its omnichannel imperative. Another example is Mt. Sinai Health System, which employs APIs to integrate legacy systems and enable a single patient view and thereby enhance patient care [1]. Elizabeth Hackenson, Schneider Electric CIO, employs an "API-first" approach involving widespread use of APIs in support of a service-oriented business model [3]. Further business applications of APIs as digital infrastructure components are illustrated in Table 1. With such novel and valuable applications, APIs are elevating digital infrastructure to be viewed as part of business strategy [34].

Table 1. Business Applications of APIs

\begin{tabular}{|l|l|}
\hline $\begin{array}{l}\text { Resource } \\
\text { sharing }\end{array}$ & $\begin{array}{l}\text { Call center headset maker exposes } \\
\text { processed audio data to customers for } \\
\text { identifying effective customer service } \\
\text { strategies. }\end{array}$ \\
\hline $\begin{array}{l}\text { Cloud } \\
\text { computing }\end{array}$ & $\begin{array}{l}\text { Integration of on-premises legacy } \\
\text { enterprise systems with new } \\
\text { cloud-based customer-relationship } \\
\text { management systems. }\end{array}$ \\
\hline $\begin{array}{l}\text { Data } \\
\text { compliance }\end{array}$ & $\begin{array}{l}\text { Open banking APIs enable banks to } \\
\text { allow customers to share data subsets } \\
\text { with other banking institutions, } \\
\text { complying with PSD2 regulations. }\end{array}$ \\
\hline $\begin{array}{l}\text { Supply } \\
\text { Chains }\end{array}$ & $\begin{array}{l}\text { Shipping firms use APIs as digital } \\
\text { infrastructure to expose capabilities such } \\
\text { as scheduling and featuring self-service } \\
\text { for supply chain partners. }\end{array}$ \\
\hline
\end{tabular}

In summary, given the importance of APIs as digital infrastructure as well as the scarcity of prior research addressing deployment challenges, we conduct an exploratory examination of the organizational deployment of APIs as digital infrastructure. We ask the following research question: What organizational challenges hinder the deployment of APIs within large organizations and thereby hamper digital service innovation?

We begin with a brief review of the literature, followed by discussion of our chosen conceptual framework, research methodology, research findings, and discussion of implications for theory and practice.

\section{Prior Research}

Research on digital infrastructure has begun to emerge as an important area of IS scholarship. Several research directions have been proposed based on the unique properties of digital infrastructure, including infrastructure qualities and development, as well as the role of, and impact on, individuals, groups, organizations, and markets [32]. The centrality of APIs to suggested research directions has also been underscored: "the ability to define an API serving as the "obligatory passage point" can be viewed as a control point both decentralizing and centralizing access, while at the same time both regulating behavior and enabling access." [32:755].

Given the new features of modern APIs and their economic significance, scholars have begun to explore boundary resources as a component of digital infrastructure (APIs being a current instantiation). Two major streams of emerging literature involve: 1) boundary resources in the context of platform ecosystems, and 2) boundary resources in the context of artifact and organizational perspectives (Table 2).

\subsection{Boundary Resources: Platform Ecosystem Perspectives}

Boundary resources play a critical role in platform ecosystems - marketplaces of producers and consumers supported by digital infrastructure and standards [31, 33]. In the context of platform ecosystems, boundary resources act as control points for digital resource sharing. As such, APIs have been viewed from network mapping perspectives to elucidate platform ecosystem structure and dynamics. For example, researchers have analyzed the structural networks and clusters formed by public APIs in use [7, 14].

Shifting from the structural to the dynamic, researchers have also analyzed how API boundary resources support management of tensions in the Apple iOS service platform in terms of innovation and control [6]. The Android mobile platform has also been the subject of analysis regarding the role of boundary resources in exploiting and defending digital platforms [18]. In sum, an emerging literature has analyzed boundary resources in the context of platform ecosystems, contributing important knowledge to their role in digital resource sharing.

\subsection{Boundary Resources: Artifact \& Organizational Perspectives}

Boundary resources have also been examined as technical artifacts, including development frameworks, documentation, security, design, performance, and usage. For example, researchers seek to enhance API operational reliability by analyzing automated test case generation [2]. Another example is API usability in support of the correct use of APIs to avoid cascading errors and potential system failure [30]. Boundary resources have also been analyzed as a means by which to share and extend design capabilities [25]. 
From an organizational perspective, researchers have examined APIs from a service innovation perspective to identify three API archetypes that support service development: integrator, free data provider, and mediator [34]. Also from a service design perspective, APIs have been discussed as important aspects of infrastructural layers as boundary resources [9] within the layered modular architecture [36]. Researchers have also begun to examine APIs from innovation and strategic perspectives, focusing on impacts and implications for organizations [15].

Effective deployment of boundary resources within firms is necessary for leveraging service innovation, platform business models, and other forms of digital innovation. However, deployment has not been a focus of prior research [27]. This provides the key motivation for our study [32:753]: "Research on the nature, design, and evolution of these infrastructures should start by capturing the sociotechnical infrastructural dynamics of specific cases and the study context." This study provides a modest step towards addressing this call to research in the context of boundary resources.

\section{Table 2. Research on Boundary Resources} (APIs) as Digital Infrastructure

\begin{tabular}{|l|l|}
\hline & Example \\
\hline $\begin{array}{l}\text { Platform } \\
\text { Peosystem }\end{array}$ & $\begin{array}{l}\text { Mapping and visualizing boundary } \\
\text { resource structure [7, 14]. Mobile } \\
\text { software platform dynamics [6, 18]. }\end{array}$ \\
\hline $\begin{array}{l}\text { Artifact } \\
\text { Perspectives }\end{array}$ & $\begin{array}{l}\text { APIs as a technical artifact, including } \\
\text { development frameworks, } \\
\text { documentation, security, design, } \\
\text { performance, and usage [2, 25]. }\end{array}$ \\
\hline $\begin{array}{l}\text { Organizational } \\
\text { Perspectives }\end{array}$ & $\begin{array}{l}\text { Service innovation, layered modular } \\
\text { architectures, strategic and } \\
\text { economic implications [9, 15, 26, 34, } \\
\end{array}$ \\
\hline
\end{tabular}

\section{Conceptual Background}

We adopt an existing framework of digital infrastructure to inform our study and facilitate interpretation of our findings. The framework involves two key paradoxes (or tensions) of digital infrastructure: the paradox of change and the paradox of control [32]. It is important to address these tensions because change in large organizations is particularly disruptive and must be managed to exercise greater control over the diffusion of APIs.

The paradox of change refers to the need to provide both stability (transparent and stable environment for others to leverage) and flexibility (opportunities for extension and expansion to grow functionality over time). For example, the Blue Button API supports interoperability of U.S. Medicare claims data between different systems, and must navigate the paradox of change by providing sufficient stability to its different stakeholders to support development and ensure reliable operations while enabling continued expansion of its services. This can cause failure modes when features or data sources are deprecated in new APIs to make way for the new.

The paradox of control refers to the tension between centralized versus distributed control of the API. For example, an organization such as Instagram retains centralized control of its APIs, which can impact the incentive of its various stakeholders to continue their collaboration with the platform. In contrast, financial regulations concerning open banking have developed a hybrid control mechanism by which different institutions can have a say in API governance in the realm of PSD2.

The change and control paradoxes have dimensions spanning different levels (individual, group, organizational, infrastructure, development, market, etc.). The paradoxes inform our findings, as we discuss below.

\section{Research Methodology}

Given the rapidly emerging and evolving research context, we adopted an exploratory revelatory multi-case study approach to shed light on new phenomena that have not been the subject of scholarly attention [35]. Based on this choice, our data collection and analysis methodology followed a three-stage approach.

\subsection{Stage 1 - Explore milieu of APIs-in-use}

In the first stage, our objective was to prepare for data collection and analysis by understanding the broad scope of API use contexts across a range of industries from different perspectives. To this end, we searched multiple sources including ProgrammableWeb.com, which has an extensive list of public APIs as well as related articles and documentation. We also searched vendor and consulting reports, such as those written by Apigee and Gartner. In addition, we interviewed a senior executive of a leading API management platform to gather additional market perspectives and insights (Mulesoft). Knowledge gained informed the second stage of data collection involving our case 
protocol comprising the type of company, the type of respondent, and open-ended questions.

\subsection{Stage 2 - Specify research protocol}

Regarding firm type, we chose firms in two different industries for two reasons. First, we observed on ProgrammableWeb.com and vendor reports that a significant percentage of firms using and consuming APIs lie outside technology industries. Moreover, non-technology firms have higher technical debt, a culture less compatible with constant change, and organizational structures that may not align with APIs. This drove our choice of firms outside the technology sector, i.e., to provide a more holistic and revealing picture of both API challenges and opportunities. Second, numerous APIs are published in the banking (777), education (516), and healthcare (152) industries (ProgrammableWeb.com). We thus sought potential case sites in these three industries and identified one each in healthcare and education (the authors could not identify a case in financial services).

The first case site is a regional healthcare organization focusing on efficient patient care while maintaining operational effectiveness. At a middle stage of adoption, the healthcare organization has successfully deployed several APIs internally and externally to support enhanced patient care and drive operational efficiencies. The second case site is a large post-secondary educational institution at an early stage of API adoption. A few different middleware products are being employed, a small set of APIs has been written, and some of those (as well as external APIs) are being employed in production operations.

Table 3. Respondents \& Interview Duration

\begin{tabular}{|c|l|c|}
\hline & Title & Duration $^{*}$ \\
\hline A1 & Web Development Manager & 35 \\
\hline A2 & IT Infrastructure Manager & 38 \\
\hline A3 & Senior Vice President & 64 \\
\hline B1 & Service Owner Learning Mgmt. & 53 \\
\hline B2 & Director of Web Applications & 68 \\
\hline B3 & Developer & 48 \\
\hline B4 & Bl Team Lead & 46 \\
\hline B5 & Data Integration / API Manager & 31 \\
\hline B6 & Senior Development Officer & 41 \\
\hline B7 & Finance Executive & 42 \\
\hline & & 466 \\
\hline
\end{tabular}

Note: A: Healthcare, B: Education. *Duration in minutes.
Regarding respondent type, we learned in Stage 1 about the important role played by technical and non-technical employees from different organizational levels in API development and operations. This drove our choice of a broad and inclusive respondent set containing business and technical staff within each case site (Table 3). Based on the size of each case site as well as some preliminary knowledge of their API usage, we interviewed three members of the first case site and seven members of the second case site.

\section{Table 4. Interview Questions \& Data Coding}

\begin{tabular}{|c|}
\hline $\begin{array}{l}\text { Background } \\
\text { Role in the organization, day to day responsibilities, } \\
\text { tenure, mission, etc. }\end{array}$ \\
\hline $\begin{array}{l}\text { Technology Context } \\
\text { Business Staff: Major IT services and/or data sources } \\
\text { that enable your group /unit to achieve its objectives, } \\
\text { benefits, challenges. Technical Staff: Key technical } \\
\text { platforms (systems, stacks, etc.) that enable your group } \\
\text { /unit to achieve its objectives, benefits, challenges. }\end{array}$ \\
\hline $\begin{array}{l}\text { API Perspectives } \\
\text { Business Staff: Understanding of APIs, consumer or } \\
\text { producer of APIs (both), main benefits, challenging or } \\
\text { difficult aspects. Technical Staff: Understanding of APIs, } \\
\text { usage of APIs, direct involvement in their production or } \\
\text { consumption, difficult aspects. }\end{array}$ \\
\hline $\begin{array}{l}\text { API Adoption } \\
\text { Adopt drivers, centralized/decentralized approach, } \\
\text { planning process, etc. }\end{array}$ \\
\hline $\begin{array}{l}\text { API Implementation } \\
\text { Implementation process, focus on any one particular } \\
\text { area, unexpected challenges, role of vendor } \\
\text { relationship, important stakeholders, overall sense for } \\
\text { API implementation. }\end{array}$ \\
\hline $\begin{array}{l}\text { API Impacts and Implications } \\
\text { Impacts or implications of API implementation } \\
\text { unexpected impacts, advice for other organizations }\end{array}$ \\
\hline $\begin{array}{l}\text { Data Coding } \\
\text { Text segment labels ("data privacy") based on } \\
\text { transcribed interviews grouped into categories ("data } \\
\text { governance"), shared, and refined; axial coding uses } \\
\text { context and causal conditions to group categories into } \\
\text { themes ("ensuring data integrity"). }\end{array}$ \\
\hline
\end{tabular}

Interview questions were driven by our research question [35] and included five areas: respondent specifics, the organizational context, API perspectives, API adoption, API implementation, and API implications (Table 4). For each respondent, we shared our semi-structured interview protocol beforehand and received consent to record each conversation. Seven of ten interviews were conducted by the authors in person with the respondent, with the remaining done via online conferencing software. 


\subsection{Stage 3 - Analysis of qualitative data}

All interviews were transcribed, coded, and synthesized following standard practice in five steps [11]. First, we conducted initial text segment tagging using concept labels (e.g., data privacy). Then, we grouped concept labels into categories (e.g., data governance) and shared and refined the categories among research team members. Third, we used axial coding to identify the context and causal conditions associated with each category. Fourth, after several rounds of iteration, we grouped categories into themes (e.g., ensuring data integrity) and developed structural narratives driven by our conceptual framework (paradoxes of change and control), linking themes, to categories, to concept labels, to text segments. At the conclusion of the three-stage research methodology lasting roughly six months, four salient themes emerged, two of which tied directly to the paradoxes.

\section{Results}

\subsection{Ensuring Data Integrity: Paradox of Control}

As a data sharing mechanism, APIs impact data integrity (completeness, consistency, and accuracy) [21]. Firms in the midst of API deployment confront these impacts in a variety of contexts, which can impact deployment speed and effectiveness.

On the one hand, APIs can enhance data integrity, for example, by enabling a granular and time-based audit trail of which employee is accessing which resource. APIs can also enable real-time data visibility and automated data pulls, which decreases errors in industries such as logistics by enabling instantaneous freight quotes. As one of our technical respondents underscored regarding such benefits of APIs: "The security and the audit abilities of API's just skyrocket."

On the other hand, the positive impacts of APIs on data integrity are not automatic and require significant changes in data governance and business processes. Regarding data governance, a business manager underscored its importance to effective deployment of APIs as follows: "I think the data governance is the biggest obstacle." A specific data integrity and accuracy issue raised was in regards to transferring data among systems, including systems of record. As elaborated by a business respondent: "The enterprise system is the book of record, and so I need attention to be paid because then you don't want there to be unintended consequences that they just start doing all their work off the API" - in other words, this process may threaten the integrity of the data in the ledger if there is no longer a single source of truth.

Implications of APIs for data integrity in the realm of business processes were emphasized in several contexts. A business respondent put it this way: "The current method is not totally manual but it's not totally automated. I have my control to eyeball it and look through it ... whereas this API exchange or ESB [Enterprise Service Bus] exchange, you know, feels like it's just data flying everywhere and I didn't have a chance to look at it and it's so out of control." Security and its implications for data accuracy were also underscored by respondents. For example, a business leader emphasized that: "To the extent there's security roles in our enterprise system and the API has none, we've asked, 'How does that work?' So it introduces new risk, so they say, 'I promise I'm not gonna let anybody access it that shouldn't.' So that's fine, I guess, but then you've distributed your responsibility for controlling the data security, and what are best practices for that?"

Overall, though APIs may feature affordances for enhancing data integrity, such affordances require new governance mechanisms and are not necessarily understood by key decision makers, as emphasized by a technical respondent: "I try to present [enhanced security and audit capabilities] back to the data governance managers, helping them understand what API's are, but it's still a hard sell." The observed managerial challenge related to data integrity is thus to understand the data integrity implications of APIs and how to enable associated process and governance changes that incorporate the new features of APIs.

In essence, the paradox of control must be managed within the organization to ensure that local data owners are comfortable with data integrity that might be controlled by units outside of their control that specify API processes and policies. Management must overcome the perception that API-enabled approaches automatically increase risk, while digital leadership must appreciate the perception of the negative impact of APIs on data integrity (Table 5).

\subsection{Aligning Incentives: Digital Commons Problem}

The second key finding resulting from our qualitative empirical analysis of respondent interviews concerns the lack of incentives regarding producing APIs. Developers will naturally seek third-party APIs 
to enhance their productivity. However, they may not always embrace the idea (often coming from management) of developing an API that will be consumed by others inside and outside the firm (including documentation regarding authentication, error messages, resources exposed, terms of use, etc.). Amazon famously overcame this issue by mandating that all resources be shared via APIs, specifically, that all teams must have the ability to expose resources to other teams and other companies, without exception. Respondents in our case organizations in the process of deploying APIs were not subject to such a strict mandate, with implications for API production.

\section{Table 5: Key Findings}

Ensuring Data Integrity - Paradox of Control
APIs affect data completeness, consistency, and
accuracy. Observed issues include governance
approaches, information security, compliance,
distributed risk models, training, and process change.
Aligning Incentives - Digital Commons Problem
Incentive issues when there is no incentive to produce
APIs, only to consume those produced by others, or
when certain integration approaches are preferred by
management but not developers.
Developing Shared Understanding - Asymmetric
Knowledge Problem
APIs mean different things to different organizational
stakeholders. Lack of shared understanding inhibits
effective adoption and diffusion.
Managing API Producers - Paradox of Change
New forms of supplier management challenges emerge
in the API domain, given a lack of formal SLAs and
unclear enforceability (e.g., upgrades that do not
provide backward compatibility).

One technical respondent suggested that the lack of benefits to exposing resources inhibits the development of APIs: "if I were asked to do some data integration or publish some data for a certain group, I will be thinking whether I should publish it to the API directory? What benefit do I get instead of standing up another web server?" A technical lead echoed this sentiment, empathizing with the overworked nature of development as another inhibitor: "they viewed it as extra work because they weren't consuming them, so it wasn't part of their architecture to build reusable things for them to use and then share publicly. It was an add-on, and nobody wants extra work, right?" Both of these comments illustrate root causes underlying one type of misaligned incentive concerning APIs: management wants more APIs developed but developers don't see immediate benefits to their own productivity in developing them.
Another incentive issue concerns the choice of a particular integration approach when multiple options are possible. Management perceives the value of certain approaches, which may differ from those preferred by developers: "We verbalized it as - you won't have to use these CSV files anymore. It'll get easier. The exchange will be live." However, developers may opt to use approaches to satisfy their own objectives. As one technical manager put it: "We have both direct API access to [a new cloud service], and then access that's brokered through the enterprise service bus (ESB). We're definitely responsible for encouraging the use of [the ESB for auditing and control]. Yes, you can generate a token off your account and have your application actually using your account-generated API. When you leave, your account may be deprovisioned." Conflicting incentives and choices thus lead to inefficiency and risk of system interruption.

In sum, various types of incentive issues were revealed with respect to the use of APIs for data integration and resource exposure. One framework within which to view these issues is as a digital commons problem in which developers are happy to use already existing APIs but are reluctant to create them if they do not benefit directly from their use. The result is "overgrazing" and reduced efficacy [24]. The managerial challenge is to incentivize and promote a particular workflow from a set of viable options that is beneficial from a long-term organizational standpoint, but that may not be perceived to be optimal from a short-term business unit or individual perspective.

\subsection{Developing Shared Understanding: Asymmetric Knowledge Problem}

Traditional information systems such as enterprise resource planning (ERP) systems have clearly defined functionality, a common user interface, and clear system boundaries. In contrast, APIs have varying functionality and often have no conventional user interface. Moreover, APIs often span organizational boundaries, raising new challenges such as a phenomenon that has been referred to as "the politics of APIs" [20]. As a result, though respondents were selected for their knowledge of some aspect of APIs (e.g, application development, financial reporting, or infrastructure operations), we expected some variation in how respondents perceive APIs. To gauge perceptions of APIs, develop a baseline terminology for subsequent questions, and minimize potential miscommunication, we asked respondents about their 
perceptions of APIs. Our prior belief was that respondents would share a common and reasonably valid sense for the functionality, features, and basic operations of APIs. This was not the case.

Respondents provided a broad and diverse range of answers to our query about how they perceive APIs, including "IT infrastructure," "marketing tool," "data," "revenue source," and "connecting system." Moreover, the connotation of API also varied, from something that is a useful tool to something that should be treated with suspicion. For example, a business leader who viewed APIs as a connecting system suggested that APIs as a general concept are too risky and immature to be implemented in production.

Overall, what we thought would be a minor question revealed a critically important and unexpected feature of APIs. While it makes sense that technical and business stakeholders would view APIs through their own respective "points of entry," such as data, middleware, governance, tasks, and incentives, the degree of variation within and across such stakeholder groups was significant. Without a shared understanding of API basics, it is difficult to fathom how organizations can conduct effective deployment.

A managerial challenge is thus to overcome an asymmetric knowledge problem in which digital infrastructure features and affordances are understood by a limited few, but stakeholders without such knowledge must make related strategic decisions. Managers must determine how best to educate and inform staff members as to the features and functionality of APIs to enable a shared understanding of possibilities and risks and thereby overcome the asymmetric knowledge problem. This mechanism might prove important beyond the current context as ever more complicated digital infrastructures and systems emerge in the Fourth Industrial Revolution [23].

\subsection{Managing API Producers: Paradox of Change}

When APIs are consumed across organizational boundaries, new challenges arise. For example, an API producer may update an API's functionality to output data in a slightly different way. This in turn affects the operation of the calling application. If it is not upgraded to align with the change, errors ensue. This (not uncommon) scenario raises managerial issues related to service level agreements as well as tacit verbal agreements, both in terms of how changes will occur (frequency, versioning, etc.) as well as how they will be communicated (communication modes, lead time, etc.)

For example, in 2013 Netflix stopped its open API program that had been used by developers to test and operate applications such as film streaming aggregator services, disrupting downstream development. Another example is ridesharing app Uber, which began with the Google Maps API, moved to another map service due to pricing and service uncertainty, then switched back to Google Maps API. As smart home technology proliferates, upstream API producers may be increasingly responsible for outages in the realms of home security and home automation, with significant implications for downstream product and service providers [22].

The fourth finding concerns a strong pattern of case study respondents describing facets and nuances pertinent to this issue, including API reliability, versioning, and overall service levels. In particular, a pattern of concern involved transparent communication and standards for managing how APIs are upgraded by producers both internal and external to the company, with respondents wondering about risks to operations when APIs are used in production.

For example, a business unit director, responsible for provisioning a business-critical cloud-based service throughout the organization, emphasized the need for tight collaboration and communication of the API producer to the user organization in terms of lead times when changes and upgrades are made. One issue concerns the time it takes to prepare for an API change: "You have to give us enough time because we have to change the application, we have to test the application, and deploy it." Beyond operational concerns, the need for overall governance and service level maturity were also underscored: "So helping them to understand the level of maturity that they need to have for these APIs that we develop enterprise services on top of ... we need that level of maturity around change management."

Another area of concern was more general and concerned the reliance on outsourced API developers. For example, one respondent voiced concerns about the use of an outside API developer and what may arise when changes are needed: "If I had to reach back out to that vendor to get him to make API edits, or anything like that, you know, just the fact that it was outsourced, makes that a little difficult. If we ever did need to get a hold of him, you know, we're at his beck and call at this point." Such issues of hold up and a general concern regarding navigating such uncertainty were raised by respondents in both organizations. 
This managerial challenge thus relates to managing producers and owners of APIs, including such issues as versioning, transparency, and joint understanding of service level agreements. This is a broad managerial issue related to the paradox of change, though not limited to it. Moreover, it extends beyond APIs, but has new dimensions particular to the API context. For example, in a long-term supplier arrangement, both written contracts and the power of buyer or supplier dictate what happens when things (inevitably) don't go according to plan. But in the case of APIs, power relationships are less clear, precedent is emerging, and issues are often dealt with on the technology side rather than the business side, with unintended and sometimes value destroying consequences.

\section{Discussion}

Qualitative empirical analysis of interview data collected from respondents across two large organizations revealed a response to our research question regarding API deployment challenges. Four challenges were identified and described using rich data collected within two organizations: ensuring data integrity, aligning incentives, developing shared understanding, and managing API producers.

In addition, while not sufficiently numerous to constitute patterns, our data also revealed a number of additional challenges. These included the choice and use of API management platforms, the value of analytics provided by popular APIs such as Twilio for messaging, ad hoc versus systematic identification of existing APIs to meet emerging business requirements, cloud computing as an API adoption incentive, APIs as a cybersecurity attack surface, uncertainty around API use versus enhancing an existing system, the presence of an API directory as an innovation catalyst, and developing new governance mechanisms to balance operational efficiency with customer satisfaction, such as throttling limits.

Respondents also shared their own sense of how various issues they face might be overcome. For example, regarding developing a shared understanding of APIs, one respondent shared their own journey: "I needed to be educated in the case of the ESB [Enterprise Service Bus] of how it was similar and different to what I already knew... I benefited immensely by having a white-boarding session of here's my piece of data. It's starting in system x. Now it's traveling over into this middle where it's now gone over into system y. What happens over there in those systems? What kind of manipulation could happen?"

From a scholarly perspective, and connecting back to existing literature reviewed earlier, our study contributes to the organizational perspective research stream within the digital infrastructure literature (Table 2). Our findings provide insights into the paradoxes or tensions of control and change. In terms of control, APIs as controlling boundary resources created a tension in that some employees viewed APIs as ceding control of data privacy and integrity to technical personnel. The implication was a reticence to adopt APIs as they perceived risk to data integrity. In this sense, our study suggests that the implications of the paradoxes (or tensions) can impact the ability of the entire organization to adopt boundary resources as a form of digital infrastructure. This yields a question for future research: how is culture shaped and reshaped towards data and resource sharing and consumption within and outside the firm? In other words, how might tensions and paradoxes of control be overcome?

The second paradox or tension concerning change has an analogy to supply chain management. In this case, management of suppliers (vetting, service level agreements, etc.) may be adapted to the boundary resource context. Moreover, as boundary resources proliferate, new ways of tracking hundreds or thousands of boundary resources built by external firms may also be adapted. For example, a graph database may be used to visualize the entire structure of boundary resources being called and delivered in real time - akin to a network graph showing the status of physical global supply chain goods in transport.

Beyond the two tensions, other potential research questions arise. First, how do firms develop new sources of revenue via APIs? What new structures are required, what new incentive schemes are necessary, and how to price API services (revenue sharing, free, etc.)? Second, what new governance structures are required to support a thriving API-based organization? How can transparency be achieved, security ensured, and sufficient flexibility allowed to enable generativity?

\section{Conclusion}

In the fourth industrial revolution, machine-to-machine information and resource sharing are accelerating and functionally changing. A key implication is that formerly clear boundaries of physical, digital, and biological systems are blurring [23]. API boundary resources are a critical underlying 
component of digital infrastructure that enables such business change and transformation. While this study provided a modest first step to addressing knowledge gaps regarding deployment challenges, further research is needed to understand how firms are transitioning to API enablement to support new business models for internal integration and external resource monetization. Such research will provide a foundation for models and frameworks supporting effective digital infrastructure management in the fourth industrial revolution.

\section{References}

[1] Aleryani, R., "How Mount Sinai Health System is redefining healthcare delivery using MuleSoft", MuleSoft Blog, 2017.

https://blogs.mulesoft.com/biz/industries/mount-sinai-redef ines-healthcare-delivery/

[2] Arcuri, A., "RESTful API Automated Test Case Generation with EvoMaster", ACM Transactions on Software Engineering and Methodology 28(1), 2019, pp. 3:1-3:37.

[3] Boulton, C., "How Schneider Electric Grooms 'Digital Experts' to Spark Transformation”, CIO, 2018.

[4] Broadbent, M., P. Weill, and D. St. Clair, "The Implications of Information Technology Infrastructure for Business Process Redesign", MIS Quarterly 23(2), 1999, pp. 159-182.

[5] Constantinides, P., O. Henfridsson, and G.G. Parker, "Introduction-Platforms and Infrastructures in the Digital Age", Information Systems Research 29(2), 2018, pp. 381-400.

[6] Eaton, B., S. Elaluf-Calderwood, and C. Sorensen, "Distributed Tuning of Boundary Resources: The Case of Apple's iOS Service System”, MIS Quarterly 39(1), 2015, pp. 217-243.

[7] Evans, P.C., and R.C. Basole, "Revealing the API Ecosystem and Enterprise Strategy via Visual Analytics", Communications of the ACM 59(2), 2016, pp. 26-28.

[8] Fink, L., and S. Neumann, "Gaining Agility through IT Personnel Capabilities: The Mediating Role of IT Infrastructure Capabilities", Journal of the Association for Information Systems 8(8), 2007, pp. 440-462.

[9] Ghazawneh, A., and O. Henfridsson, "Governing Third-Party Development Through Platform Boundary Resources", ICIS 2010 Proceedings, (2010).

[10] Giraldo-Mora, J., M. Avital, and J. Hedman, "Development Dynamics of Digital Infrastructure and Organization: The Case of Global Payments Innovation”,

\section{Acknowledgment}

We thank leaders of respondent organizations for making key staff available for interviews, without which, this research would not have been possible.

Proceedings of the 40th International Conference on Information Systems (ICIS), AIS Electronic Library (AISeL) (2019), 1456.

[11] Green, J., K. Willis, E. Hughes, et al., "Generating Best Evidence from Qualitative Research: the Role of Data Analysis", Australian and New Zealand Journal of Public Health 31(6), 2007, pp. 545-550.

[12] Hanseth, O., and K. Lyytinen, "Design Theory for Dynamic Complexity in Information Infrastructures: the Case of Building Internet", Journal of Information Technology 25(1), 2010, pp. 1-19.

[13] Henfridsson, O., and B. Bygstad, "The Generative Mechanisms of Digital Infrastructure Evolution", MIS Quarterly 37(3), 2013, pp. 907-931.

[14] Huhtamäki, J., R. Basole, K. Still, M. Russell, and M. Seppänen, "Visualizing the Geography of Platform Boundary Resources: The Case of the Global API Ecosystem", HICSS - 50, (2017).

[15] Iyer, B., and M. Subramaniam, "Are You Using APIs to Gain Competitive Advantage?", Harvard Business Review, 2015.

[16] Jensen, T., Y.-H. Tan, and N. Bjørn-Andersen, "Unleashing the IT Potential in the Complex Digital Business Ecosystem of International Trade: The Case of Fresh Fruit Import to European Union", BLED 2014 Proceedings, (2014).

[17] Jussila, J., J. Kukkamaki, M. Mantyneva, and J. Heinisuo, "Open Data and Open Source Enabling Smart City Development: A Case Study in Hame Region", Technology Innovation Management Review 9(9), 2019, pp. 25-34.

[18] Karhu, K., R. Gustafsson, and K. Lyytinen, "Exploiting and Defending Open Digital Platforms with Boundary Resources: Android's Five Platform Forks", Information Systems Research 29(2), 2018, pp. 479-497.

[19] Koutsikouri, D., R. Lindgren, O. Henfridsson, and D. Rudmark, "Extending Digital Infrastructures: A Typology of Growth Tactics", Journal of the Association for Information Systems 19(10), 2018, pp. 1001-1019. 
[20] Lane, K., "Politics of APIs", API Evangelist, 2014.

[21] Markarian, J., "Data Integrity Challenges in Manufacturing”, Pharmaceutical Technology 40(7), 2016, pp. 50-54.

[22] McCarthy, K., "Smart bulbs turn dumb: Lights out for Philips as Hue API goes dark", The Register, 2018. https://www.theregister.co.uk/2018/06/01/philips_hue_api_ dark/

[23] Melville, N., and L. Robert, "The Fourth Industrial Revolution - Towards a New Paradigm of Technology-Enabled Change", 2020. University of Michigan

[24] Melville, N., A. Stevens, R. Plice, and O. Pavlov, "Unsolicited Commercial Email: Empirical Analysis of a Digital Commons", International Journal of Electronic Commerce, 2006.

[25] Mohagheghzadeh, A., and F. Svahn, "Shifting Design Capability to Third-Party Developers: An Affordance Perspective on Platform Boundary Resources", AMCIS 2016 Proceedings, 2016.

[26] Ofoeda, J., and R. Boateng, "Institutional Effects on API Development and Integration in Developing Countries: Evidence from Ghana", AMCIS 2018 Proceedings, (2018).

[27] Ofoeda, J., R. Boateng, and J. Effah, “Application Programming Interface (API) Research: A Review of the Past to Inform the Future", International Journal of Enterprise Information Systems 15(3), 2019, pp. 76-95.

[28] Queiroz, M., P. Tallon, T. Coltman, and R. Sharma, "Digital Infrastructure, Business Unit Competitiveness, and Firm Performance Growth: The Moderating Effects of Business Unit IT Autonomy", Proceedings of the 53rd Hawaii International Conference on System Sciences, (2020).

[29] Raivio, Y., S. Luukkainen, and S. Seppälä, “Towards Open Telco - Business Models of API Management Providers", 2011 44th Hawaii International Conference on System Sciences, (2011), 1-11.

[30] Rauf, I., E. Troubitsyna, and I. Porres, "A Systematic Mapping Study of API Usability Evaluation Methods", Computer Science Review 33, 2019, pp. 49-68.

[31] de Reuver, M., C. Sørensen, and R.C. Basole, "The Digital Platform: A Research Agenda", Journal of Information Technology Impact 33(2), 2018, pp. 124-135.

[32] Tilson, D., K. Lyytinen, and C. Sørensen, "Research Commentary-Digital Infrastructures: The Missing IS Research Agenda", Information Systems Research 21, 2010, 748-759.

[33] Van Alstyne, M.W., G.G. Parker, and S.P. Choudary, "Pipelines, Platforms, and the New Rules of Strategy",
Harvard Business Review 94, 2016, pp. 54 - 62.

[34] Wulf, J., and I. Blohm, "Service Innovation through Application Programming Interfaces - Towards a Typology of Service Designs", International Conference on Information Systems, (2017).

[35] Yin, R.K., Case Study Research, Design and Methods, Sage Publications, Thousand Oaks, CA, 2009.

[36] Yoo, Y., O. Henfridsson, and K. Lyytinen, “The New Organizing Logic of Digital Innovation: An Agenda for Information Systems Research", Information Systems Research 21(4), 2010, pp. 724-735. 